ORIGINAL ARTICLE

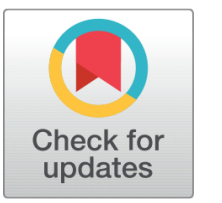

OPEn ACCESS

Received: 27-03-2020

Accepted: 24-04-2020

Published: 04-06-2020

Editor: Dr. Natarajan Gajendran

Citation: Singh VK, Srivastava S, Vibhu I (2020) Study of transmittance spectra for quaternary periodic structure of 1D photonic crystal for s-wave filter. Indian Journal of Science and Technology 13(15): 1580-1588. https://doi.org/ 10.17485/IJST/V13i15.24

* Corresponding author. Vishal Kumar Singh

Research scholar, Department of Physics, Integral University, Lucknow, 226026, U.P, India.

Tel.: 91-945-323-2699

singh87vishal@gmail.com

Funding: None

Competing Interests: None

Copyright: @ 2020 Singh, Srivastava, Vibhu. This is an open access article distributed under the terms of the Creative Commons Attribution License, which permits unrestricted use, distribution, and reproduction in any medium, provided the original author and source are credited.

Published By Indian Society for Education and Environment (iSee)

\section{Study of transmittance spectra for quaternary periodic structure of 1D photonic crystal for s-wave filter}

\author{
Vishal Kumar Singh ${ }^{1 *}$, Seema Srivastava ${ }^{2}$, Isht Vibhu ${ }^{3}$ \\ 1 Research scholar, Department of Physics, Integral University, Lucknow, 226026, U.P, India. \\ Tel.: 91-945-323-2699 \\ 2 Associate Professor, Department of Physics, Integral University, Lucknow, 226026, U.P, \\ India \\ 3 Associate Professor, Department of Physics, Y.D.P.G college, Lakhimpur Kheri, 262701, U.P, \\ India
}

\section{Abstract}

Objectives: To study the propagation of electromagnetic waves through the four periodic continuous layers with different refractive indices and spectral analysis with normalized frequency with respect to transmittance and dispersion. Methods: By considering the assumptions the theoretical analysis has been done. Methodology has taken from quantum mechanical treatment of an electron in a potential well with boundary conditions. The dispersion relation is taken from Bloach wave concept. The calculation is taken for arbitrary structure of different refractive indices. The electric field has connected with the periodic 1-D layered structure. The electromagnetic wave as form incident wave, the transmitted wave and reflected wave have been derived using the transmission matrix method. Findings: In theoretical analysis, it is found that the transmittance spectra increasing with continuous increase refractive index. The analysis has been done for a s-polarized or transverse electric mode (TE mode) wave. Properties of these crystals are different from the ordinary materials. Applications: It works as s- polarized filter and blocks p-polarized wave. Calculation has taken for TE mode configuration.

Keywords: Photonic crystals; Electromagnetic waves; Periodic layered structure

\section{Introduction}

With proper application of potential, motion of electron in a dielectric can be controlled. The speed of electron in a circuit has certain limitations. To improve the speed of information, photons can be considered as carrying agents, which travel with the speed of light. It is difficult to control the motion of photons than an electron. The photon is related to the light and light is an electromagnetic wave. The electromagnetic wave has electric as well as the magnetic field. These two different fields are responsible for the interaction with materials. Electric field interacts with material's atom while the interaction of magnetic field is normally weak ${ }^{(1)}$. Photonic crystals are emerging in the field of Photonics and are used in different areas such as to control the propagation of light, 
controlling of signals for a specific frequency. Passing information from one place to another is faster through electromagnetic wave than electronic signals in photonic crystals. The idea of ligament model for light has led in the last 40 years in optics and optical technology to a completely new direction that deals with periodically dielectric media and the light propagation busy in it. ${ }^{(2)}$ The novel materials, which have extremely interesting properties, were given the name Photonic Crystal (PC). As crystal is a periodic arrangement of atoms or molecules in space ${ }^{(3)}$, like that photonic crystals are the periodic arrangement of dielectric materials. Its periodicity is of the order of a wavelength. It can be constructed by the periodic arrangement of dielectric-dielectric or dielectric-metal materials. The composition of metal and a dielectric layer is said to be metallodielectric photonic crystals ${ }^{(4)}$. It is found with properties like perfect reflectance, used as anti-reflecting coatings. Refractive index defines the properties of a material and is given in terms of permittivity $(\varepsilon)$ and permeability $(\mu)$ of the medium as $n=\sqrt{\varepsilon \mu}$. Photonic crystals provide gap, which is photonic band-gap, which controls the propagation of waves. The regular multiplication of a wave inside the crystal provides band-gap. Band-gap is the frequency range for which the propagation of photons is prohibited inside the photonic crystal $^{(5)}$. Photonic crystals have some specific photonic band structures. It has properties like, pass band, stop band and defect states, that is, some frequencies are allowed and some forbidden. Researchers are continuously trying to construct photonic crystals as per specific need. In this process, there are various techniques have been developed to fabricate photonic crystals for the practical application. The laser direct writing method, vertical deposition method and the laser holographic lithography method are used for the fabrication of photonic crystals. Eli Yablonovitch and Sajeev Jhon independently proposed the concept of photonic crystal in $1987^{(6)}$. The first assumption to control the propagation of light by a one dimensional periodic structure was given by Igor A. et. al. ${ }^{(7)}$. Ho K. M. et. al. proposed the band-gap structure for FCC lattices. The construction of periodic optical dielectric metamaterials that affects the motion of photons is called as photonic crystals. These are macroscopic periodic or non-periodic artificial structures govern by both their cellular architecture and chemical composition with specific properties ${ }^{(8)}$. Metamaterial structure exists negative index dielectric material of refraction. The negative refraction has been experimentally verified by Valanju et al. ${ }^{(9)}$. In the present work, theoretical analysis of the four layer periodic photonic crystal structure has been done. The medium is assumed to be isotropic and lossless. Electromagnetic wave incident on the surface of crystal then reflection, refraction and transmission of waves occurs. Continuously increase in refractive index, angle of refraction should decrease in a regular way. The wave will propagate in a particular direction. Transmittance should increase. The transmittance of the electromagnetic wave propagation has exactly calculated by the transfer matrix method (TMM) ${ }^{(10)}$. This gives an idea that light is guided by the materials. By considering the assumptions the theoretical analysis has done. The results have plotted by the calculations. Methodology has taken from quantum mechanical treatment of an electron in a potential well with boundary conditions ${ }^{(11)}$. The dispersion relation is taken from Bloach wave concept. ${ }^{(12)}$ The analysis has done for a s-polarized or transverse electric mode (TE mode) wave.

\section{Theoretical analysis}

Now we are considering a $1 \mathrm{D}$ periodic structure in which propagation of an electromagnetic wave from the left to the right side along the positive $\mathrm{X}$-axis direction and the launching medium is homogeneous along incident and transmitted directions.

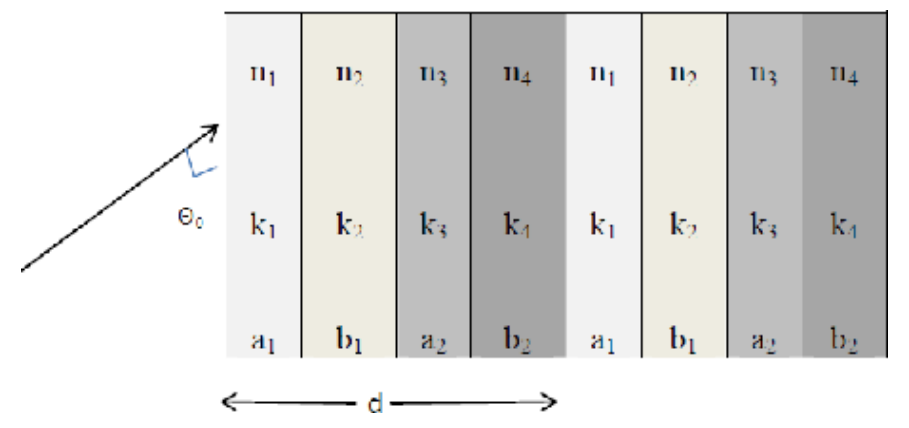

Fig 1. Periodic layered 1-D structure

The electric field has connected with the periodic 1-D layered structure. The electromagnetic wave as form incident wave, the transmitted wave and reflected wave has been derived using the transmission matrix method. The Maxwell's equations of electromagnetic wave and interface boundary conditions gives the electric fields. The derivatives of equation show the continuity of the electric field at the interfaces of each $1 \mathrm{D}$ layer. By mathemetical calculation, in $\mathrm{n}^{\text {th }}$ layer structure, the amplitude of the 
wave related to the transfer matrix as:

$$
\left(\frac{A_{n-1}}{B_{n-1}}\right)=\left(\begin{array}{ll}
m_{11} & m_{12} \\
m_{21} & m_{22}
\end{array}\right)\left(\frac{A_{n}}{B_{n}}\right)
$$

Where $\mathrm{m}_{11}, \mathrm{~m}_{12}, \mathrm{~m}_{21}$ and $\mathrm{m}_{22}$ are the elements of transfer matrix. ${ }^{(13)}$. Transfer matrix elements $\mathrm{m}_{11}, \mathrm{~m}_{12}, \mathrm{~m}_{21}$ and $\mathrm{m}_{22}$ are :

$$
\begin{aligned}
& m_{11}=\frac{1}{4}\left(e^{i k_{1} a} \cdot e^{i k_{3} a_{1}}\left(\sin \left(k_{2} b\right)\left(\frac{i k_{2}}{k_{3}}+\frac{i k_{3}}{k_{4}}\right)+\cos \left(k_{2} b\right)\left(\frac{k_{2}}{k_{4}}+1\right)\right\} \times\left(\sin \left(k_{4} b_{1}\right)\left(\frac{i k_{1}}{k_{2}}+\frac{i k_{4}}{k_{1}}\right)+\cos \left(k_{4} b_{1}\right)\left(\frac{k_{4}}{k_{2}}+1\right)\right\}\right] \\
& +\frac{1}{4}\left(e^{i k_{1} a} \cdot e^{-i k_{3} a_{1}}\left(\sin \left(k_{2} b\right)\left(\frac{i k_{2}}{k_{3}}-\frac{i k_{3}}{k_{4}}\right)-\cos \left(k_{2} b\right)\left(\frac{k_{2}}{k_{4}}-1\right)\right\} \times\left(\sin \left(k_{4} b_{1}\right)\left(\frac{i k_{1}}{k_{2}}-\frac{i k_{4}}{k_{1}}\right)-\cos \left(k_{4} b_{1}\right)\left(\frac{k_{4}}{k_{2}}-1\right)\right\}\right] \\
& m_{12}=-\frac{1}{4}\left(e^{-i k_{1} a} \cdot e^{i k_{3} a_{1}}\left(\sin \left(k_{2} b\right)\left(\frac{i k_{2}}{k_{3}}+\frac{i k_{3}}{k_{4}}\right)+\cos \left(k_{2} b\right)\left(\frac{k_{2}}{k_{4}}+1\right)\right\} \times\left(\sin \left(k_{4} b_{1}\right)\left(\frac{i k_{1}}{k_{2}}-\frac{i k_{4}}{k_{1}}\right)+\cos \left(k_{4} b_{1}\right)\left(\frac{k_{4}}{k_{2}}-1\right)\right\}\right] \\
& -\frac{1}{4}\left(e^{-i k_{1} a} \cdot e^{-i k_{3} a_{1}}\left(\sin \left(k_{2} b\right)\left(\frac{i k_{2}}{k_{3}}-\frac{i k_{3}}{k_{4}}\right)-\cos \left(k_{2} b\right)\left(\frac{k_{2}}{k_{4}}-1\right)\right\} \times\left(\sin \left(k_{4} b_{1}\right)\left(\frac{i k_{1}}{k_{2}}+\frac{i k_{4}}{k_{1}}\right)-\cos \left(k_{4} b_{1}\right)\left(\frac{k_{4}}{k_{2}}+1\right)\right\}\right] \\
& m_{21}=-\frac{1}{4}\left(e^{i k_{1} a} \cdot e^{i k_{3} a_{1}}\left(\sin \left(k_{2} b\right)\left(\frac{i k_{2}}{k_{3}}-\frac{i k_{3}}{k_{4}}\right)+\cos \left(k_{2} b\right)\left(\frac{k_{2}}{k_{4}}-1\right)\right\} \times\left(\sin \left(k_{4} b_{1}\right)\left(\frac{i k_{1}}{k_{2}}+\frac{i k_{4}}{k_{1}}\right)+\cos \left(k_{4} b_{1}\right)\left(\frac{k_{4}}{k_{2}}+1\right)\right\}\right] \\
& -\frac{1}{4}\left(e^{i k_{1} a} \cdot e^{-i k_{3} a_{1}}\left(\sin \left(k_{2} b\right)\left(\frac{i k_{2}}{k_{3}}+\frac{i k_{3}}{k_{4}}\right)-\cos \left(k_{2} b\right)\left(\frac{k_{2}}{k_{4}}+1\right)\right\} \times\left(\sin \left(k_{4} b_{1}\right)\left(\frac{i k_{1}}{k_{2}}-\frac{i k_{4}}{k_{1}}\right)-\cos \left(k_{4} b_{1}\right)\left(\frac{k_{4}}{k_{2}}-1\right)\right\}\right] \\
& m_{22}=\frac{1}{4}\left(e^{-i k_{1} a} \cdot e^{i k_{3} a_{1}}\left(\sin \left(k_{2} b\right)\left(\frac{i k_{2}}{k_{3}}-\frac{i k_{3}}{k_{4}}\right)+\cos \left(k_{2} b\right)\left(\frac{k_{2}}{k_{4}}-1\right)\right\} \times\left(\sin \left(k_{4} b_{1}\right)\left(\frac{i k_{1}}{k_{2}}-\frac{i k_{4}}{k_{1}}\right)+\cos \left(k_{4} b_{1}\right)\left(\frac{k_{4}}{k_{2}}-1\right)\right\}\right] \\
& +\frac{1}{4}\left(e^{-i k_{1} a} \cdot e^{-i k_{3} a_{1}}\left(\sin \left(k_{2} b\right)\left(\frac{i k_{2}}{k_{3}}+\frac{i k_{3}}{k_{4}}\right)-\cos \left(k_{2} b\right)\left(\frac{k_{2}}{k_{4}}+1\right)\right\} \times\left(\sin \left(k_{4} b_{1}\right)\left(\frac{i k_{1}}{k_{2}}+\frac{i k_{4}}{k_{1}}\right)-\cos \left(k_{4} b_{1}\right)\left(\frac{k_{4}}{k_{2}}+1\right)\right\}\right]
\end{aligned}
$$

Where $k_{1}, k_{2}, k_{3}$ and $k_{4}$ are the wave propagation vectors in $a_{1}, a_{2}, b_{1}$ and $b_{2}$ thickness of the layers respectively, which is given by the following relation:

$$
k_{j}=\left(\left(\frac{n_{j} \omega}{c}\right)^{2}-\beta^{2}\right]^{\frac{1}{2}}=\left(\frac{\omega}{c}\right) n_{j} \cos \theta_{j}, \quad \theta_{j}=\sin ^{-1}\left(\frac{n_{0} \cdot \sin \theta_{0}}{n_{j}}\right)
$$

where $\beta$ is the $\mathrm{z}$-component of the wave vector. $j=1,2,3$ and 4 respectively.

The Bloch wave with period $d\left(=a_{1}+a_{2}+b_{1}+b_{2}\right)$, by the condition as:

$$
E_{K}(x+d)=E_{K}(x)
$$

we can write the dispersion relation as:

$$
K=\cos ^{-1} \frac{1}{d}\left(m_{11}+m_{22}\right]
$$

The band gap argument corresponds to undefined complex solutions of a real dispersion relation. ${ }^{(14)}$

By the use of the Chebyshev Identity ${ }^{(15)}$, the transfer matrix for $\mathrm{N}$ number of periodic layers is calculated as:

$$
M=\left(\begin{array}{ll}
M_{11} & M_{12} \\
M_{21} & M_{22}
\end{array}\right)=\left(\begin{array}{ll}
m_{11} & m_{12} \\
m_{21} & m_{22}
\end{array}\right)^{N}=\left(\begin{array}{cc}
m_{11} U_{N-1}-U_{N-2} & m_{12} U_{N-1} \\
m_{21} U_{N-1} & m_{22} U_{N-1}-U_{N-2}
\end{array}\right)
$$

where

$$
U_{N}=\frac{\sin [(N+1) K d]}{\sin K d}
$$

and the transmittance of the wave is given by:

$$
T=\left(\frac{1}{M_{11}}\right)^{2}=\left(\frac{1}{m_{11} U_{N-1}-U_{N-2}}\right)^{2}=\left(\frac{1}{1-\left(m_{21}\right)^{2}(\sin N K d / \sin K d)^{2}}\right]
$$

By the use of equation (11), we get the transmittance of the wave and by the use of equation (8) get the photonic band gap. 


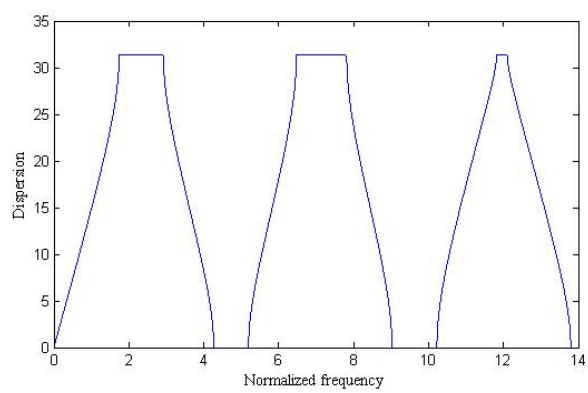

Fig 2. Normalized frequency and dispersion relation, $n_{1}=1.0, n_{2}=1.50, n_{3}=2.0, n_{4}=2.50, a_{1}=0.045, b_{1}=0.015, a_{2}=0.030, b_{2}=$ $0.010, \theta=45$

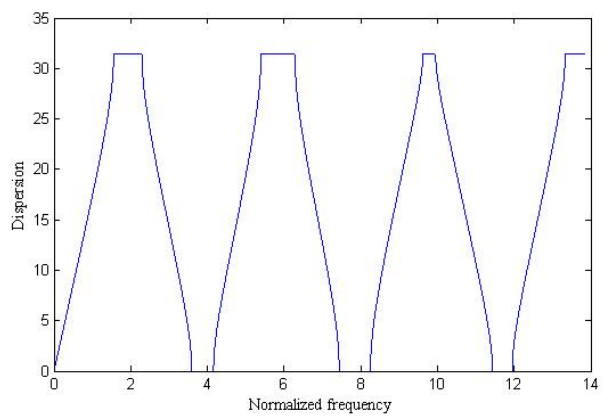

Fig 3. Normalized frequency and dispersion relation, $n_{1}=1.25, n_{2}=1.75, n_{3}=2.25, n_{4}=2.75, a_{1}=0.045, b_{1}=0.015, a_{2}=0.030, b_{2}=$ $0.010, \theta=45$.

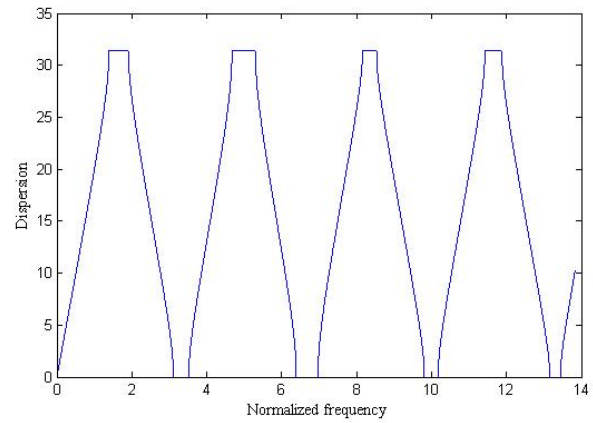

Fig 4. Normalized frequency and dispersion relation, $n_{1}=1.50, n_{2}=2.0, n_{3}=2.50, n_{4}=3.0, a_{1}=0.045, b_{1}=0.015, a_{2}=0.030, b_{2}=$ $0.010, \theta=45$.

\section{Results}

By the use of equations (6) and (8), the Figures 2, 3 and 4 are plotted for dispersion relation with normalized frequency. It is found that the band-gap appears for some frequencies. Propagation wave in this frequency range is prohibited. The bandgap tunes with change in refractive indices of medium. It has observed that band-gap shifts towards lower frequencies so the propagation of waves for smaller frequencies will be prohibited.

Figures 4, 5, 6 and 7 show transmittance spectra for variation of angle of incidence and refractive indices. Here observed some peaks at 0,30 and $60^{\circ}$ it means for selected frequency transmittance will be maximum. As the increase in angle of incidence, the peak shifts towards higher frequency so transmittance can obtain for higher frequencies analytically.

Figures 8, 9, 10 and 11 shows that transmittance has been observed. In Figures 8 and 10 do not show a good transmission, here only at a particular frequency the transmission is observed. 


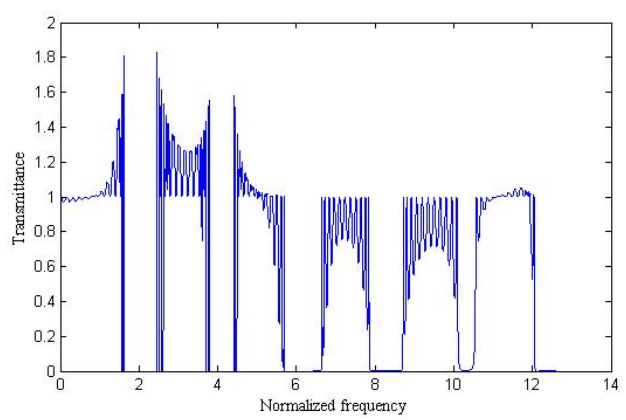

Fig 5. Figures for normalized frequency and transmittance, $n_{1}=1.0, n_{2}=1.50, n_{3}=2.0, n_{4}=2.50, a_{1}=0.045, b_{1}=0.015, a_{2}=$ $0.030, b_{2}=0.010, \theta=0$

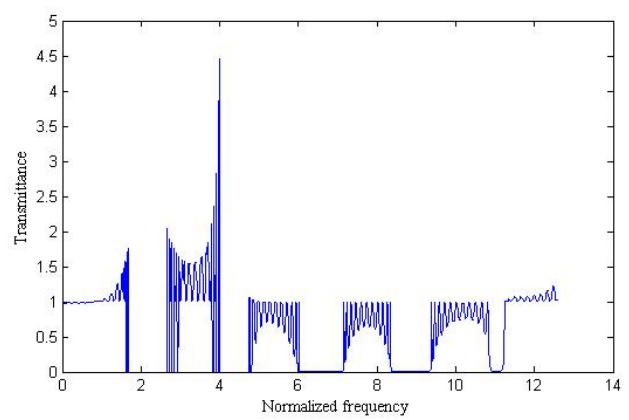

Fig 6. Figures for normalized frequency and transmittance, $n_{1}=1.0, n_{2}=1.50, n_{3}=2.0, n_{4}=2.50, a_{1}=0.045, b_{1}=0.015, a_{2}=$ $0.030, b_{2}=0.010, \theta=30$.

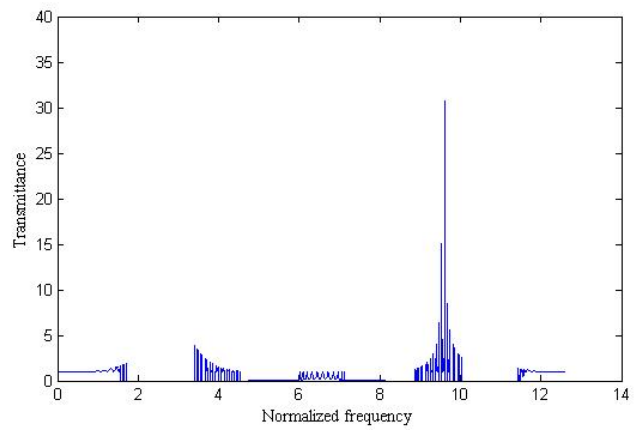

Fig 7. Figures for normalized frequency and transmittance, $n_{1}=1.0, n_{2}=1.50, n_{3}=2.0, n_{4}=2.50, a_{1}=0.045, b_{1}=0.015, a_{2}=$ $0.030, b_{2}=0.010, \theta=60$.

In Figures 12, 13, 14 and 15 found a good transmittance but Figure 11 is good only for a particular frequency. Figures 12, 13, 14 and 15 are transmittance spectra for a four layer structure. The regions with zero transmittance founded in all. There are some regions that show zero transmittance. In that region perfect reflectance will observe. The propagation wave is prohibited in these regions. It shows the photonic band-gap. 


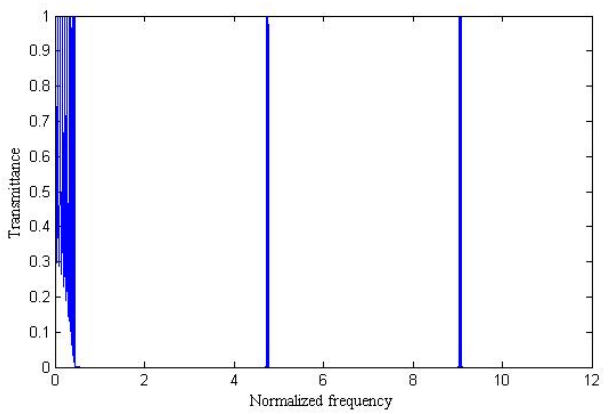

Fig 8. Figures for normalized frequency and transmittance, $n_{1}=1.0, n_{2}=1.50, n_{3}=2.0, n_{4}=2.50, a_{1}=0.045, b_{1}=0.015, a_{2}=$ $0.030, b_{2}=0.010, \theta=89$.

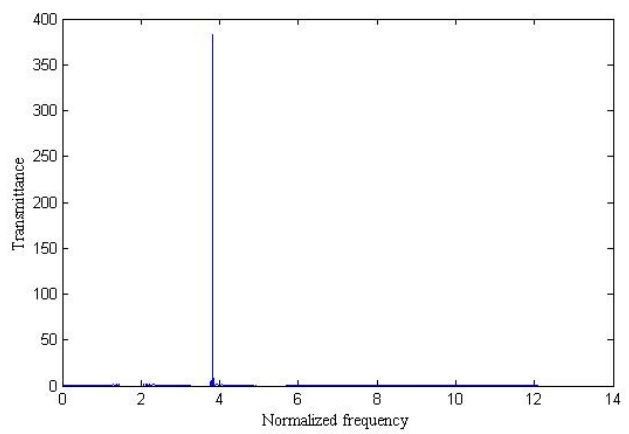

Fig 9. Figures for normalized frequency and transmittance, $n_{1}=1.25, n_{2}=1.75, n_{3}=2.25, n_{4}=2.75, a_{1}=0.045, b_{1}=0.015, a_{2}=$ $0.030, b_{2}=0.010, \theta=0$.

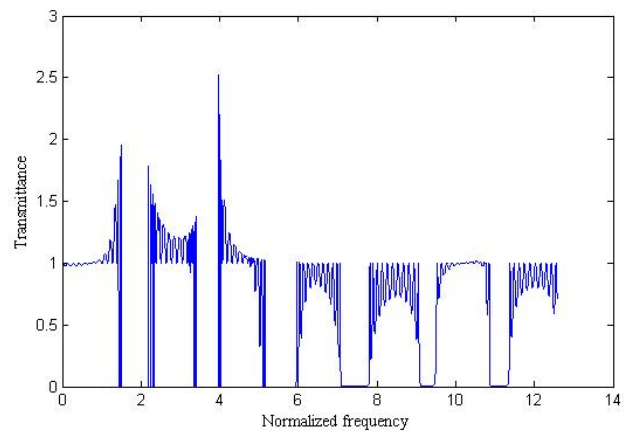

Fig 10. Figures for normalized frequency and transmittance, $n_{1}=1.25, n_{2}=1.75, n_{3}=2.25, n_{4}=2.75, a_{1}=0.045, b_{1}=0.015, a_{2}=$ $0.030, b_{2}=0.010, \theta=30$. 


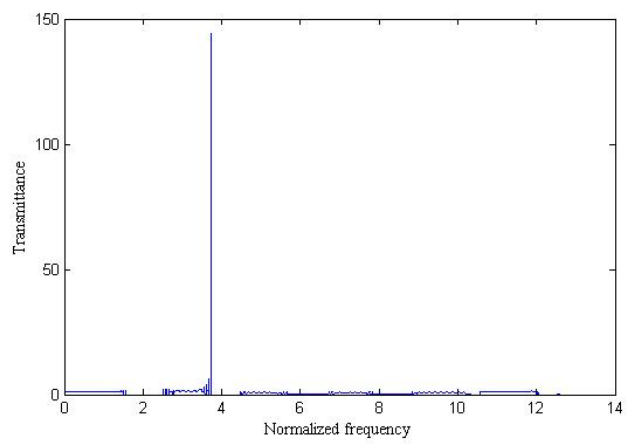

Fig 11. Figures for normalized frequency and transmittance, $n_{1}=1.25, n_{2}=1.75, n_{3}=2.25, n_{4}=2.75, a_{1}=0.045, b_{1}=0.015, a_{2}=$ $0.030, b_{2}=0.010, \theta=60$.

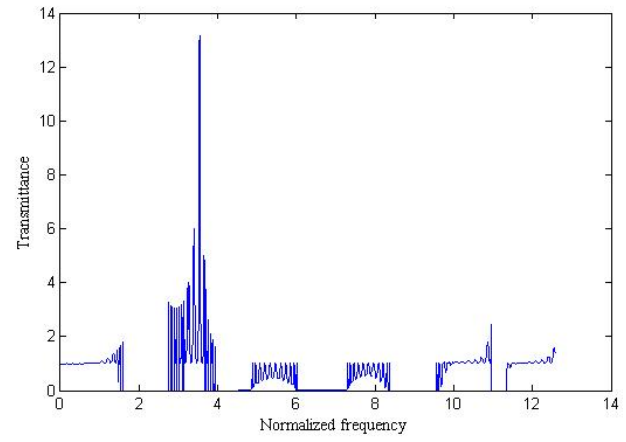

Fig 12. Figures for normalized frequency and transmittance, $n_{1}=1.25, n_{2}=1.75, n_{3}=2.25, n_{4}=2.75, a_{1}=0.045, b_{1}=0.015, a_{2}=$ $0.030, b_{2}=0.010, \theta=89$.

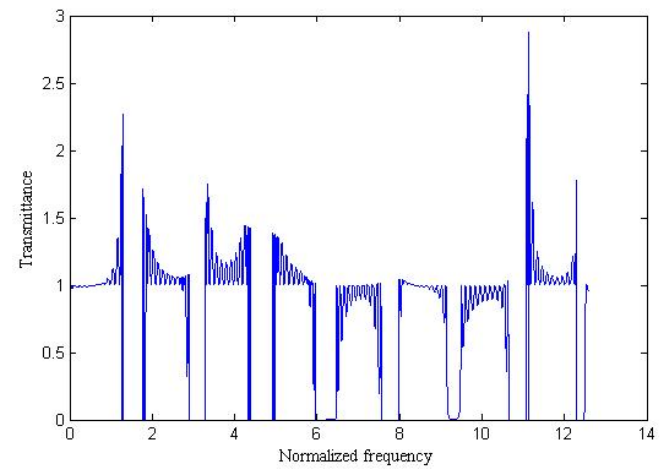

Fig 13. Figures for normalized frequency and transmittance, $n_{1}=1.50, n_{2}=2.0, n_{3}=2.50, n_{4}=3.0, a_{1}=0.045, b_{1}=0.015, a_{2}=$ $0.030, b_{2}=0.010, \quad \theta=0$. 


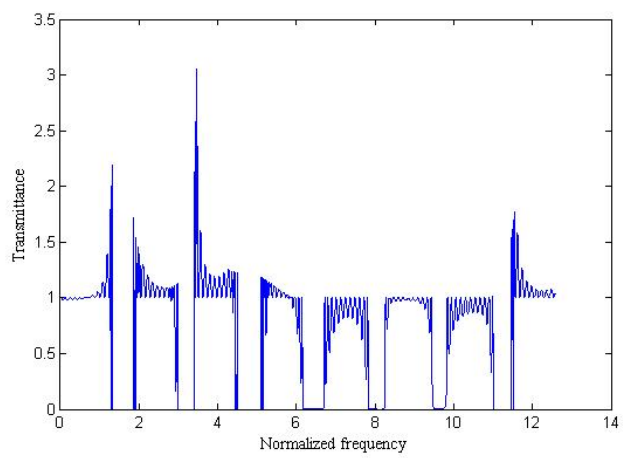

Fig 14. Figures for normalized frequency and transmittance, $n_{1}=1.50, n_{2}=2.0, n_{3}=2.50, n_{4}=3.0, a_{1}=0.045, b_{1}=0.015, a_{2}=$ $0.030, b_{2}=0.010, \theta=30$.

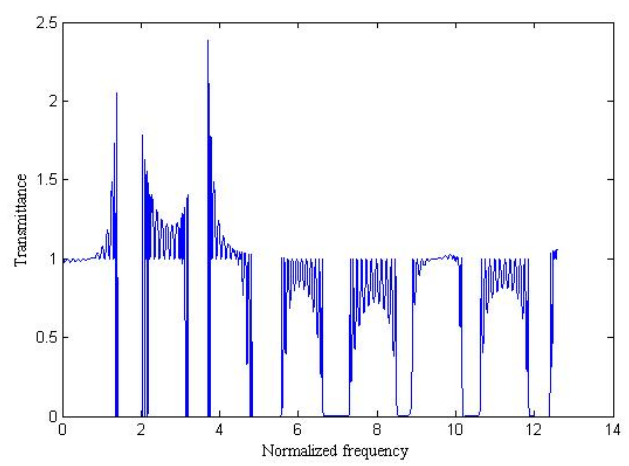

Fig 15. Figures for normalized frequency and transmittance, $n_{1}=1.50, n_{2}=2.0, n_{3}=2.50, n_{4}=3.0, a_{1}=0.045, b_{1}=0.015, a_{2}=$ $0.030, b_{2}=0.010, \theta=60$.

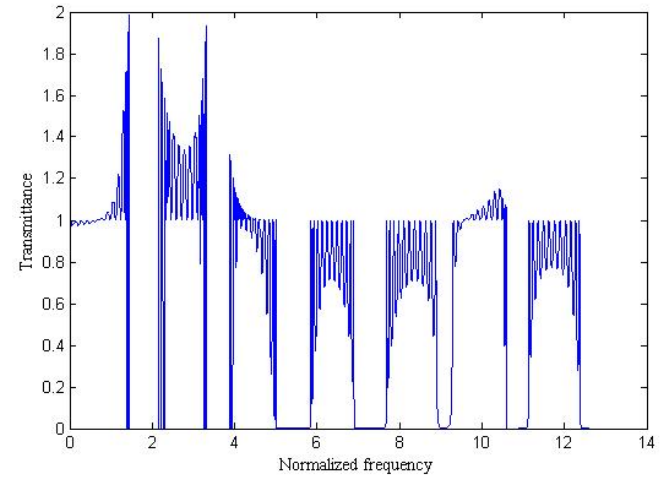

Fig 16. Figures for normalized frequency and transmittance, $n_{1}=1.50, n_{2}=2.0, n_{3}=2.50, n_{4}=3.0, a_{1}=0.045, b_{1}=0.015, a_{2}=$ $0.030, b_{2}=0.010, \theta=89$. 


\section{Conclusion}

From the results and analysis, it is found that the structure appears with band-gap by the increase in refractive index at different refractive incidences. For some angles with a constant refractive index of materials, transmittance found to be maximum for a particular frequency. These structures can be used for the selection of a specific frequency. The materials lie between the considered refractive indices should show the same behavior as obtained. It is for s-polarized wave so; it works as s-polarized filter and completely stops the p-polarized wave.

\section{Acknowledgement}

Authors are thankful to the research and development of integral University, Lucknow for providing the research facilities and manuscript communication number IU/R\&D/2020-MCN000811.

\section{References}

1) Awasthi ASK, Mishra A, Malaviya U, Ojha SP. Wave propagation in a one-dimensional photonic crystal with metamaterial. Solid State Communications. 2009;149(33-34):1379-1383. Available from: https://doi.org/10.1016/j.ssc.2009.05.017.

2) Amiri SIS, Razalli. Bin Azzuhri, Muhammad Arif Jalil, "Introduction to Photonics: Principles and the Most Recent Applications of Microstructures". Micromachines. 2018.

3) Joannopoulos JD, Johnson SG, Winn JN, Meade RD. Photonic Crystals Molding The Flow of Light. Copyright Princeton University Press. 2008. Available from: https://dl.acm.org/doi/book/10.5555/1628775.

4) Gong Q. CRC Press. 2014. Available from: https://doi.org/10.1201/b15654.

5) Chhajed S, Design. Fabrication, and Characterization of Optical Coatings Made of Low- and Tunable-Refractive-Index Materials. Rensselaer Polytechnic Institute Troy, New York. 2010. Available from: https://search.proquest.com/openview/444ec9f54de9abb23b11809efec0845a/1?pq-origsite=gscholar\& $\mathrm{cbl}=18750 \&$ diss $=\mathrm{y}$.

6) Yablonovitch E. Inhibited Spontaneous Emission in Solid-State Physics and Electronics. Physical Review Letters. 1987;58(20):2059-2062. Available from: https://dx.doi.org/10.1103/physrevlett.58.2059. doi:10.1103/physrevlett.58.2059.

7) Sukhoivanov IA, Guryev IV. Photonic Crystals. In: and others, editor. Physics and Practical Modeling;vol. 152. Springer-Verlag Berlin Heidelberg. 2009. Available from: https://doi.org/10.1007/978-3-642-02646-1.

8) Metamaterials. In: Cui TJ, Smith D, Liu R, editors. Theory, Design, and Applications. Springer. 2010. Available from: https://doi.org/10.1007/978- 1-44190573-4.

9) Srivastava R, Shyam KBT, Ojha PSP. Reply to comment on "Negative refraction in 1D photonic crystals'. Solid State Communications. 2011;151:579-581. Available from: https://dokumen.tips/documents/reply-to-comment-on-negative-refraction-in-1d-photonic-crystals.html. doi:10.1016/j.ssc.2010.12.026.

10) .. Available from: http://assets.press.princeton.edu/chapters/s8695.pdf.

11) Schwarz K. Computation of Materials Properties at the Atomic Scale. 2015. Available from: https://doi.org/10.5772/59108.

12) Pochi, Yeh. Optical Waves in Layered Media. John Wiley and Sons. 1988.

13) Mbakop FK, Djongyang N, Raïdandi D. One-dimensional TiO2/SiO2 photonic crystal filter for thermophotovoltaic applications. Journal of the European Optical Society-Rapid Publications. 2016;12(1):23-23. Available from: https://dx.doi.org/10.1186/s41476-016-0026-4.

14) Vila J, Pal RK, Ruzzene M, Trainiti G. 2017. Available from: https://arxiv.org/pdf/1702.01728.pdf.

15) Mamri B, Barkat O. Design of a Selective Filter Based on One-dimensional Superconductor Photonic Crystal. Journal of Superconductivity and Novel Magnetism. 2019;32(11):3397-3405. Available from: https://dx.doi.org/10.1007/s10948-019-5118-0. 